\title{
Could Irisin Levels be Affected by Physical Activity in Patients with Schizophrenia?
}

\author{
Gamze Erzin', Olga Güriz ${ }^{1}$, Ali Yalçındağ${ }^{2}$, Akfer Kahıloğulları', Sibel Örsel ${ }^{1}$ \\ Departments of ${ }^{1}$ Psychiatry and ${ }^{2}$ Biochemistry, Health Sciences University Dışkapı Yıldırım Beyazıt Training and Research Hospital, Ankara, Turkey
}

Objective: The aim of this study was to explore the effect of physical activity and metabolic parameters on irisin levels in patients with schizophrenia and healthy controls.

Methods: Ninety-six patients with schizophrenia and 63 healthy controls comprised the study population. The participants were separated into three groups: inactive, low activity, and sufficiently active according to International Physical Activity Questionnaire short form (IPAQ-SF). We measured irisin levels using Enzyme linked immunosorbent assay. We also calculated exercise levels by using the IPAQ-SF for each individual. The independent samples $t$ test was used in the data analysis to compare irisin levels according to the activity levels of the patients with schizophrenia and controls.

Results: The levels of irisin were higher in the healthy controls $(p<0.001)$ compared to schizophrenia groups. When the activity levels of the schizophrenia and healthy control groups were compared, the irisin levels of the low activity and sufficiently active groups with schizophrenia were found to be lower than those of the low activity and sufficiently active groups in the healthy controls (respectively $p=0.014 ; p<0.001$ ).

Conclusion: Irisin levels could be affected by physical activity and these results must be supported with new studies.

KEY WORDS: Exercise; Schizophrenia; Biomarkers; Adipokines.

\section{INTRODUCTION}

Irisin is a recently defined myokine [1] with a structure of peptide formed by 112 amino-acids and it's release is induced by exercise [2]. Irisin is formed by proteolytic cleavage of fibronectin type 3 domain containing factor protein 5 (FNDC5) $[1,3,4]$. The formation of FNDC5 is promoted by physical activity in muscle and can be converted to irisin by transcriptional co-activator peroxisome proliferator-activated receptor (PPAR)-gamma coactivator 1-alpha (PGC1 $\alpha)[1]$.

As shown in the literature irisin has also central effects [5]. It is found that irisin has a role in neurogenesis and a protective role against neuronal damage caused by oxida-

Received: August 22, 2020 / Revised: November 5, 2020 Accepted: November 8, 2020

Address for correspondence: Gamze Erzin

Department of Psychiatry, Health Sciences University Dışkap1

Ylldırım Beyazıt Training and Research Hospital, Ömer

Halisdemir Boulevard, Altındağ, Ankara 06110, Turkey

E-mail: gamze.erzin@gmail.com

ORCID: https://orcid.org/0000-0001-8002-5053 tive stress [6]. Irisin and FNDC5 enhance neuronal BDNF expression [7]. Also, irisin is thought to be involved in neuronal proliferation via the STAT3 signaling pathway [8-10]. By reducing the secretion of proinflammatory cytokine-like tumor necrosis factor (TNF)-alpha and interleukin (IL)-6, and by suppressing reactive oxygen species inflammatory signal in ischemia, irisin mediates neuroprotection of physical exercise in cerebral ischemia via the Akt and ERK1/2 signaling pathways against neuronal damage [11]. All these effects of irisin suggest that it should be considered a hormone worth investigating for neuropsychiatric disorders. For example, irisin may play a role in depression by actuating the expression of BDNF through the PGC1 $\alpha /$ FNDC5 pathway [9]. Irisin promotes important processes in the nervous system, especially in the presence of neurodegenerative disorders including schizophrenia known to be linked with diminished neurogenesis $[7,8,11,12]$.

Thus, we aimed to explore the irisin levels in patients with schizophrenia and healthy controls. Our hypothesis was that irisin levels would be lower in patients with

@) This is an Open-Access article distributed under the terms of the Creative Commons Attribution Non-Commercial License (http://creativecommons.org/licenses/by-nc/4.0) which permits unrestricted non-commercial use, distribution, and reproduction in any medium, provided the original work is properly cited. 
schizophrenia compared with healthy controls and irisin levels of patients with insufficient activity levels would be lower than patients with sufficient or low activity levels.

\section{METHODS}

This study population consisted of 96 patients who were diagnosed as schizophrenia according to the Diagnostic and Statistical Manual of Mental Disorders 5th edition criteria and 63 healthy controls. Mental retardation, pregnancy, malignancy, known inflammatory disease or use of immunosuppressive agents were regarded as exclusion criteria. The ethics committee of Dışkapı Training and Research Hospital approved the study (Date: 2017; No: 39/27). Signed approval for every sample was also obtained either from the patients themselves or their first-degree relatives.

We calculated exercise levels by using the International Physical Activity Questionnaire-short form (IPAQ-SF). The participants were separated into three groups: inactive, low intensity activity, sufficiently active. At the same time, The Brief Psychiatric Rating Scale (BPRS) was used to evaluate the symptom severity of patients with schizophrenia. Laboratory tests including complete lipid parameters, fasting blood glucose, glycated hemoglobin, and insulin measurements were performed on blood samples from every subject. The waist circumferences of the subjects were measured. Body mass index (BMI) values were noted for each subject.

IPAQ-SF: IPAQ-SF was used to measure the physical activity of the participants over the last week. IPAQ-SF consists of 7 questions and the scores of each question are added at the end. As a result, IPAQ-SF gives the activity level in MET*minute/week, and participants are separated into three groups concurring to their activity levels, as described earlier [13]. The validity and reliability study of the Turkish form of the scale was shown by Saglam et al. [14].

BPRS: BPRS was used to evaluate the symptom severity of patients with schizophrenia. There are 18 questions in total and 0 to 6 scoring for each question. It is a semistructured scale used to evaluate psychotic, depressive symptoms, mannerism and posture, motor slowdown in schizophrenia, and other psychotic disorders [15]. The validity and reliability study of the Turkish form of the scale has been demonstrated [16].

\section{Blood Sampling}

After a 12-hour night fast, 5-milliliter ( $\mathrm{ml}$ ) samples of fasting blood were obtained by the participants. Blood samples were isolated from sera within 30 to 60 minutes of collection and stored at $-80^{\circ} \mathrm{C}$ until required for analysis. On the same day, IPAQ-SF was measured for each participant and BPRS was measured for each patient.

\section{Irisin Analysis}

Levels of serum irisin were specified using enzyme-linked immunosorbent assay kits (2019; BioVendor, Bmo, Czech Republic) (Cat. No.: RAG018R, Lot No: X18-047) for quantitative determination in humans. The in-work $\mathrm{CV} \%$ values were $4.863 \%$ and $6.748 \%$ for $0.678 \mu \mathrm{g} / \mathrm{ml}$ and $1.539 \mu \mathrm{g} / \mathrm{ml}$ concentrates, respectively, and the CV\% values between the runs were $9.673 \%$ and $8.027 \%$ for 0.532 $\mu \mathrm{g} / \mathrm{ml}$ and $1.145 \mu \mathrm{g} / \mathrm{ml}$ concentrates, respectively. The sensitivities were $1 \mathrm{ng} / \mathrm{ml}$ with a measurement range of $0.001-5 \mu \mathrm{g} / \mathrm{ml}$ and reference range $0.2-2 \mu \mathrm{g} / \mathrm{ml}$.

\section{Statistical Analysis}

IBM SPSS ver. 23.0 (IBM Co., Armonk, NY, USA) was used to perform statistical analyses. Independent sample $t$ test was used to analyze irisin levels, blood lipid values, and other metabolic parameters. The normality of data distribution before the analysis was evaluated using the Levene test. The normality of data distribution before the analysis was evaluated using the Levene test. Two-way independent ANOVA (2 participant groups X 3 activity groups) was used to compare the groups. Tukey's test was used for paired comparisons. Pearson correlation coefficient was used for variables with normal distribution and Spearman correlation coefficient was used for variables without normal distribution. The level of statistical significance was determined as $p<0.05$.

\section{RESULTS}

The schizophrenia group constituted $64(66.7 \%)$ males and $32(33.3 \%)$ females. The control group included 41 (65.1\%) males and $22(34.9 \%)$ females. In the schizophrenia group, $26 \%$ of the patients were working, $60.4 \%$ were not working, $2.1 \%$ were students, and $11.5 \%$ were retired. In the control group, $42.9 \%$ were working, 36.5\% were not working, $7.9 \%$ were students, and $12.7 \%$ were retired. In the schizophrenia group, $41.7 \%$ were smokers 
and $58.3 \%$ were non-smokers. In the control group, $38.1 \%$ were smokers and $61.9 \%$ were non-smokers. No significant difference was shown between the two groups with regard to smoking ( $p>0.05)$.

The mean and standard deviations BMI for the schizophrenia and control groups were $26.75 \pm 5.40 \mathrm{~kg} / \mathrm{m}^{2}$ and $26.89 \pm 3.16 \mathrm{~kg} / \mathrm{m}^{2}$ respectively (Table 1 ). According to the IFAQ-SF scores, $45.8 \%$ of the schizophrenia group was not physically active, $38.5 \%$ had low activity, and $15.6 \%$ were sufficiently physically active. It was found that $38.1 \%$ of the control group was not physically active, $38.1 \%$ had low physical activity, and $23.8 \%$ had sufficient physical activity. There was no significant difference with respect to the IFAQ-SF in either group ( $p>0.05$ ). The comparison of the laboratory finding and IFAQ-SF

Table 1. Demographic findings of samples

\begin{tabular}{lccc}
\hline \multicolumn{1}{c}{ Variable } & $\begin{array}{c}\text { Schizophrenia } \\
(\mathrm{n}=96)\end{array}$ & $\begin{array}{c}\text { Controls } \\
(\mathrm{n}=63)\end{array}$ & $p$ value \\
\hline Sex (female) & $32(33.3)$ & $22(34.9)$ & 0.84 \\
Age & $41.5 \pm 10.8$ & $41.7 \pm 13.0$ & 0.93 \\
Drug therapy & $30(31.3)$ & $\mathrm{NA}$ & - \\
Clozapine use & $66(68.7)$ & $\mathrm{NA}$ & - \\
Other antipsychotic use & $2.1 \pm 2.2$ & $\mathrm{NA}$ & - \\
Numberof hospitalizations & $14.8 \pm 8.5$ & $\mathrm{NA}$ & - \\
The duration of illness & $40(41.7)$ & $24(38.1)$ & 0.66 \\
Smoking (smoker) & $26.7 \pm 5.4$ & $26.9 \pm 3.2$ & 0.84 \\
BMI (kg/m $\left.{ }^{2}\right)$ & $21.6 \pm 8.2$ & $\mathrm{NA}$ & \\
BPRS score & & & \\
\hline
\end{tabular}

Values are presented as number $(\%)$ or mean \pm standard deviation. BMI, body mass index; BPRS, Brief Psychiatric Rating Scale; NA, not applicable. scores between the schizophrenia and control groups in the Table 2 .

No significant relationship was found between irisin and sex, comorbidities, smoking in the schizophrenia group ( $p=0.904, p=0.882, p=0.170$ ). There was no significant correlation between irisin and duration of disease, BPRS total score in schizophrenia ( $p=0.257, p=$ 0.986). Irisin levels were higher in the healthy control group ( $p<0.001$ ). In the control group, a negative correlation was found between irisin and $\mathrm{BMI}(\mathrm{r}=-0.25, p=$ $0.048)$, and there was a positive correlation between irisin and activity levels $(r=0.5, p=0.001)$.

When we divide the control group and the schizophrenia group into three according to their activity levels and compare each group with the control group; in the low-activity schizophrenia group, the irisin level is lower than in the low-activity control group; In the sufficient-active schizophrenia group, the level of irisin was found lower than the level of irisin in the sufficient-active control group ( $p=0.014 ; p<0.001$ ) (Table 3).

When the patients with schizophrenia were divided into two groups as for that use of clozapine, we did not find

Table 3. Irisin levels according to patient's activity levels

\begin{tabular}{lccr}
\hline \multicolumn{1}{c}{ Variable } & Schizophrenia & Control & $p$ value \\
\hline Non-active $(\mu \mathrm{g} / \mathrm{ml})$ & $3.7 \pm 0.8$ & $4.0 \pm 0.7$ & 0.172 \\
Low active $(\mu \mathrm{g} / \mathrm{ml})$ & $3.9 \pm 0.9$ & $4.4 \pm 0.5$ & 0.014 \\
Sufficiently active $(\mu \mathrm{g} / \mathrm{ml})$ & $3.9 \pm 0.6$ & $4.7 \pm 0.3$ & $<0.001$ \\
\hline
\end{tabular}

Values are presented as mean \pm standard deviation.

Independent samples $t$ test was used in the data analysis.

Table 2. Laboratory findings

\begin{tabular}{|c|c|c|c|}
\hline Variable & Schizophrenia $(n=96)$ & Controls $(n=63)$ & $p$ value \\
\hline Irisin $(\mu \mathrm{g} / \mathrm{ml})$ & $3.8 \pm 0.8$ & $4.3 \pm 0.6$ & $<0.001$ \\
\hline Fasting blood glucose (mg/dl) & $88(52-151)$ & $84(58-151)$ & 0.12 \\
\hline $\mathrm{HbA1c}(\%)$ & $5.6 \pm 0.5$ & $5.6 \pm 0.5$ & 0.97 \\
\hline HOMA-IR & $1.83(0.19-19.78)$ & $1.38(0.15-5.87)$ & 0.20 \\
\hline Insulin $(\mathrm{mU} / \mathrm{L})$ & $7.66(1.07-80.92)$ & $7.63(1.94-22.43)$ & 0.37 \\
\hline Waist circumference (cm) & $99.0 \pm 15.2$ & $99.4 \pm 11.6$ & 0.87 \\
\hline $\mathrm{HDL}(\mathrm{mg} / \mathrm{dl})$ & $44.1 \pm 8.8$ & $46.8 \pm 11.1$ & 0.12 \\
\hline LDL (mg/dl) & $139.5 \pm 36.8$ & $132.2 \pm 32.8$ & 0.19 \\
\hline Total cholesterol (mg/dl) & $198.9 \pm 44.9$ & $189.4 \pm 42.1$ & 0.19 \\
\hline Triglyceride (mg/dl) & $159(42-479)$ & $123(39-620)$ & 0.05 \\
\hline IPAQ-SF (MET*minute/week) & $610(0-7,550)$ & $670(0-9,600)$ & 0.24 \\
\hline
\end{tabular}

Values are presented as mean \pm standard deviation or median (minimum [min] - maximum [max]).

HOMA-IR, homeostasis model assessment of insulin resistance; HDL, high-density lipoprotein; LDL, low-density lipoprotein; IPAQ-SF, International Physical Activity Questionnaire-short form.

Independent samples $t$ test and Mann - Whitney $U$ test were used in the data analysis. 
any difference between patients using clozapine and using other antipsychotics in terms of irisin $(p=0.5)$.

When the levels of physical activity and irisin were compared with Two-way Independent ANOVA, irisin levels were found to be statistically different in schizophrenia + control and activity groups (2 schizophrenia-control group $X 3$ activity groups). In this respect, irisin levels were lower in the inactive group than in those with low or sufficient activity ( $\mathrm{F}=6.49, p=0.001)$. However, no significant difference was determined when the relationship between the irisin level and activity levels in schizophrenia but irisin levels were found to be statistically different in the control and activity groups. In this respect, irisin levels were lower in the inactive group than in those with low or sufficient activity (respectively $p=0.006 ; p=0.001$ ).

There was not any correlation between IFAQ-SF and irisin levels in schizophrenia $(p=0.28, r=0.11)$ while there was a significant correlation in the control group ( $p=$ 0.001 , spearman's rho $=0.471$ )

\section{DISCUSSION}

According to our knowledge, this is the first study to investigate the relation of irisin levels and physical activity in patients with schizophrenia. Previously, we found no differences between non-obese and nondiabetic patients with schizophrenia and controls in terms of irisin. The result of the earlier study was explained by having a small sample size and not including some metabolic parameters [17]. In the present study, we evaluated parameters that might affect levels of irisin such as physical activity levels and metabolic parameters.

In our previous study, the correlation between positive and negative syndrome scale and irisin levels were not found to be statistically significant [17]. Similarly, in this study, there was no significant correlation between irisin levels, duration of disease and BPRS total score in patients with schizophrenia. In this study, there was no difference between schizophrenia and control groups in terms of metabolic syndrome parameters and physical activity levels in the last seven days, which have been shown to affect irisin levels in previous studies. Levels of irisin, however, were found to be lower in the schizophrenia group compared to controls. In the study that measured the level of irisin in the first episode schizophrenia, the irisin level was found higher in the schizophrenia group than the control group. In the same study, the role of physical activity on the level of irisin was not investigated [18]. According to our study, physical activity may have an effect on the change in the level of irisin in the schizophrenia group. In the literature, it was found that irisin can protect neurons against oxidative damage and mitigate diabetes-induced oxidative/nitrative stresses by reducing production of superoxide and peroxynitrite, and increasing production of antioxidant enzymes including glutathione peroxidase, catalase and superoxide dismutase [18-20]. Decreasing levels of irisin in schizophrenia might be one of the reasons behind the deterioration of oxidative processes in schizophrenia.

The studies conducted on the relationship between iri$\sin$ and activity have presented different results, an increase in the levels of irisin correlated with activity levels has been obtained in various studies [21]. Dinas et al. [22] studied the acute effect of irisin on physical activity, reporting that circulating irisin levels were found to be in positive correlation with activity levels in individuals with high weekly physical activity. The level of irisin was found to be statistically higher in the low active and sufficiently active groups than in the inactive group when all participants were evaluated in our study. This result is compatible with our hypothesis that irisin levels could be affected by activity levels. A positive correlation was obtained in terms of irisin and activity levels in healthy controls.

In this study, we found that irisin was affected both by activity and found to be lower in schizophrenia independent from activity and there was no difference between the inactive schizophrenia and inactive control groups in terms of irisin levels. In a study conducted on patients with ischemic stroke, serum irisin levels were lower in patients with depression than in those without depression, and this was thought to be due to the relationship between inflammation and irisin [23]. Relationship between irisin and inflammation is shown by the positive correlation between the negative acute phase reactant retinol binding protein-4 and the anti-inflammatory adiponectin and irisin [24]. Irisin can decrease secretion of inflammatory cytokines such as TNF, IL-6, and NF- $\kappa B$ with its anti-inflammatory properties [25]. The low levels of irisin in the schizophrenia group in our study could be a result of the presence of inflammatory processes in schizophrenia. It is shown in a previous study that irisin treatment significantly reduces the levels of proinflammatory markers like 
nitrotyrosine, superoxide anion and 4-hydroxynonenal in peri-infarct brain tissues [26]. Related to these findings, irisin would find to be a therapeutic target to reduce inflammation in the brain of patients with schizophrenia in the future.

In another study including patients with Alzheimer's disease (AD), a neurodegenerative disease, it was found that increased irisin levels leads to improved cognition and $A D$ pathogenesis [27]. In our study, we didn't evaluate cognitive function in both groups. Therefore, further studies can also evaluate irisin's effect on cognition in schizophrenia and healthy controls. The irisin level in patients with the first episode schizophrenia was found to be higher than the control group [17], the reason for this result may be due to an increase in the activity levels because of positive psychotic symptoms. Therefore, it is important to evaluate the level of physical activity while comparing irisin levels in the future studies.

Irisin is formed by proteolytic cleavage of FNDC5 as a result of the activation of the transcription coactivator 1 alpha of PPAR-gamma by physical activity [7]. Therefore, there is a relationship between PPAR-gamma and irisin. In another study that compared PPAR-gamma levels in patients with schizophrenia and healthy controls, the results showed that PPAR-gamma levels were lower in the schizophrenia group [28]. Also, irisin increases neuronal BDNF expression [7]. It has been reported in meta-analyses that BDNF levels were lower in schizophrenia groups when compared with control groups [29].

The relation between PGC- $1 \alpha-$ FNDC5 and irisin-BDNF could be assumed as a pathway. Considering the relevant findings in the literature, it can be concluded that there may be a deterioration of schizophrenia in the PGC- $1 \alpha$ FNDC5/irisin-BDNF pathway. Our findings should be supported by studies evaluating this pathway as a whole.

\section{Limitations}

This situation can be evaluated more clearly in the future through longitudinal studies in which physical activity should be measured by wearable technology tools. The other limitation of this study is that the using antipsychotic can cause the alteration in levels of irisin. So, we need future studies which are planned with medication free patients with schizophrenia.

\section{Acknowledgments}

This study was supported by the Scientific Research
Committee of the Dışkapı Training and Research Hospital (funding number: 66, Date: 2017).

\section{Conflicts of Interest}

No potential conflict of interest relevant to this article was reported.

\section{Author Contributions}

Conceptualization: Gamze Erzin, Olga Güriz, Sibel Örsel. Data collection: Gamze Erzin, Olga Guriz. Formal analysis: Sibel Örsel. Funding acquisition: Gamze Erzin. Investigation: Gamze Erzin, Sibel Örsel. Methodology: Gamze Erzin, Olga Güriz, Ali Yalçındağ, Sibel Örsel. Project administration: Gamze Erzin, Sibel Örsel. Resources: Gamze Erzin. Supervision: Sibel Örsel, Akfer Kahıloğulları. Writing - original draft: Gamze Erzin, Ali Yalçındağ, Akfer Kahıloğulları, Sibel Örsel. Writing - review \& editing: Gamze Erzin, Akfer Kahıloğulları, Sibel Örsel.

\section{ORCID}

Gamze Erzin https://orcid.org/0000-0001-8002-5053

Olga Güriz https://orcid.org/0000-0001-5220-8215

Ali Yalçındağ https://orcid.org/0000-0003-1846-9248

Akfer Kahıloğulları https://orcid.org/0000-0003-2247-9609

Sibel Örsel https://orcid.org/0000-0003-0184-7038

\section{REFERENCES}

1. Boström $\mathrm{P}, \mathrm{Wu}$ J, Jedrychowski MP, Korde A, Ye L, Lo JC, et al. A PGC1- $\alpha$-dependent myokine that drives brown-fat-like development of white fat and thermogenesis. Nature 2012;481: 463-468.

2. Hecksteden A, Wegmann M, Steffen A, Kraushaar J, Morsch A, Ruppenthal S, et al. Irisin and exercise training in humans - results from a randomized controlled training trial. BMC Med 2013;11:235.

3. Teufel A, Malik N, Mukhopadhyay M, Westphal H. Frcp1 and Frcp2, two novel fibronectin type III repeat containing genes. Gene 2002;297:79-83.

4. Schumacher MA, Chinnam N, Ohashi T, Shah RS, Erickson HP. The structure of irisin reveals a novel intersubunit $\beta$-sheet fibronectin type III (FNIII) dimer: implications for receptor activation. J Biol Chem 2013;288:33738-33744.

5. Dun SL, Lyu RM, Chen YH, Chang JK, Luo JJ, Dun NJ. Irisin-immunoreactivity in neural and non-neural cells of the rodent. Neuroscience 2013;240:155-162.

6. Kim OY, Song J. The role of irisin in Alzheimer's disease. J Clin Med 2018;7:407.

7. Wrann CD, White JP, Salogiannnis J, Laznik-Bogoslavski D, Wu J, Ma D, et al. Exercise induces hippocampal BDNF 
through a PGC-10/FNDC5 pathway. Cell Metab 2013;18: 649-659.

8. Moon HS, Dincer F, Mantzoros CS. Pharmacological concentrations of irisin increase cell proliferation without influencing markers of neurite outgrowth and synaptogenesis in mouse H19-7 hippocampal cell lines. Metabolism 2013;62:11311136.

9. Ostadsharif M, Ghaedi K, Hossein Nasr-Esfahani M, Mojbafan M, Tanhaie S, Karbalaie K, et al. The expression of peroxisomal protein transcripts increased by retinoic acid during neural differentiation. Differentiation 2011;81:127-132.

10. Jung KH, Chu K, Lee ST, Kim SJ, Sinn DI, Kim SU, et al. Granulocyte colony-stimulating factor stimulates neurogenesis via vascular endothelial growth factor with STAT activation. Brain Res 2006;1073-1074:190-201.

11. Li DJ, Li YH, Yuan HB, Qu LF, Wang P. The novel exercise-induced hormone irisin protects against neuronal injury via activation of the Akt and ERK1/2 signaling pathways and contributes to the neuroprotection of physical exercise in cerebral ischemia. Metabolism 2017:68:31-42.

12. So JH, Huang C, Ge M, Cai G, Zhang L, Lu Y, et al. Corrigendum: Intense exercise promotes adult hippocampal neurogenesis but not spatial discrimination. Front Cell Neurosci 2019;13: 303.

13. Craig $\mathrm{CL}$, Marshall AL, Sjöström M, Bauman AE, Booth $\mathrm{ML}$, Ainsworth $\mathrm{BE}$, et al. International physical activity questionnaire: 12-country reliability and validity. Med Sci Sports Exerc 2003;35:1381-1395.

14. Saglam M, Arikan H, Savci S, Inal-Ince D, Bosnak-Guclu M, Karabulut E, et al. International physical activity questionnaire: reliability and validity of the Turkish version. Percept Mot Skills 2010;111:278-284.

15. Overall JE, Gorham DR. The brief psychiatric rating scale. Psychol Rep 1962;10:799-812.

16. Soykan C. Institutional differences and case typicality as related to diagnosis system severity, prognosis and treatment. Ankara:Middle East Technical University; 1990. [Thesis].

17. Erzin G, Topcuoglu C, Kotan VO, Bayram S, Fountoulakis K. Assessment of irisin, adiponectin and leptin levels in patients with schizophrenia. Endocr Metab Immune Disord Drug Targets 2018;18:163-169.

18. Zhang PF, Wang YP, Yuan XX, Tao Q, Liu YF, Pang LJ, et al. CCorrelation among serum levels of Irisin, insulin resistance and abdominal obesityin patients with first-episode schizophrenia]. Zhonghua Yi Xue Za Zhi 2019;99:520-524. Chinese.

19. Zhu D, Wang H, Zhang J, Zhang X, Xin C, Zhang F, et al. Irisin improves endothelial function in type 2 diabetes through reducing oxidative/nitrative stresses. J Mol Cell Cardiol 2015; 87:138-147.

20. Lu J, Xiang G, Liu M, Mei W, Xiang L, Dong J. Irisin protects against endothelial injury and ameliorates atherosclerosis in apolipoprotein E-Null diabetic mice. Atherosclerosis 2015; 243:438-448.

21. Kim S, Choi JY, Moon S, Park DH, Kwak HB, Kang JH. Roles of myokines in exercise-induced improvement of neuropsychiatric function. Pflugers Arch 2019;471:491-505.

22. Dinas PC, Lahart IM, Timmons JA, Svensson PA, Koutedakis $Y$, Flouris $\mathrm{AD}$, et al. Effects of physical activity on the link between PGC-1a and FNDC5 in muscle, circulating Irisin and UCP1 of white adipocytes in humans: a systematic review. F1000Res 2017:6:286

23. Tu WJ, Qiu HC, Liu Q, Li X, Zhao JZ, Zeng X. Decreased leve/ of irisin, a skeletal muscle cell-derived myokine, is associated with post-stroke depression in the ischemic stroke population. J Neuroinflammation 2018;15:133.

24. Tabak O, Simsek G, Erdenen F, Sozer V, Hasoglu T, Gelisgen $\mathrm{R}$, et al. The relationship between circulating irisin, retinol binding protein-4, adiponectin and inflammatory mediators in patients with metabolic syndrome. Arch Endocrinol Metab 2017; 61:515-523.

25. Askari $\mathrm{H}$, Rajani SF, Poorebrahim $\mathrm{M}$, Haghi-Aminjan $\mathrm{H}$, Raeis-Abdollahi E, Abdollahi M. A glance at the therapeutic potential of irisin against diseases involving inflammation, oxidative stress, and apoptosis: an introductory review. Pharmacol Res 2018;129:44-55.

26. Gaggini $M$, Cabiati $M$, Del Turco $S$, Navarra T, De Simone $P$, Filipponi $\mathrm{F}$, et al. Increased FNDC5/Irisin expression in human hepatocellular carcinoma. Peptides 2017;88:62-66.

27. Choi SH, Bylykbashi E, Chatila ZK, Lee SW, Pulli B, Clemenson $\mathrm{GD}$, et al. Combined adult neurogenesis and BDNF mimic exercise effects on cognition in an Alzheimer's mouse model. Science 2018;361:eaan8821.

28. Martínez-Gras I, Pérez-Nievas BG, García-Bueno B, Madrigal JL, Andrés-Esteban E, Rodríguez-Jiménez R, et al. The anti-inflammatory prostaglandin 15d-PGJ2 and its nuclear receptor PPARgamma are decreased in schizophrenia. Schizophr Res 2011;128:15-22.

29. Green MJ, Matheson SL, Shepherd A, Weickert CS, Carr VJ. Brain-derived neurotrophic factor levels in schizophrenia: a systematic review with meta-analysis. Mol Psychiatry 2011; 16:960-972. 